\title{
Medicinal \& Analytical Chemistry International Journal
}

\section{Men's Condomination from Incontinence}

\section{Golovkin BG*}

Department of Urology, City Clinical Hospital, Russia

*Corresponding author: Golovkin BG, Department of Urology, City Clinical Hospital No.4, Ekaterinburg, Russia, Tel: +79126414746; E-mail: gbg1940@mail.ru

\section{Introduction}

The construction of the male condom is proposed, which excludes mechanical damages of the penis from contact with its walls. To do this, the condom has a spherical extension without a joint, and the fastening is carried out using a rubber ring or rope and a ring on the tube connecting it to the urine receiver. This condom can be used to collect urine in the urine during incontinence and other urological diseases.

The invention relates to medical technology and can be used in urology as a urine collector for diseases of the genitourinary system (incontinence, incontinence). The usual male condom is a rubber condom, the wide end of which is provided with a rim of thicker rubber and is worn on the penis. The narrow end of the condom is connected on one side with a plastic tube with a urine collecting device, and on the other hand it goes into the expanding bell-shaped part of the thickened rubber (bell). The rim is connected by a tube of fine rubber with a bell with a connecting seam. A fixing device, if used, is an adhesive tape half the width of which is glued to the upper edge of the condom, and the other half is glued to the skin of the penis, or an adhesive layer is applied from the inside of the condom walls, and the strap is worn on the body or to the foot of the patient firm "COLOPLAST", the company "Urotsel").

\section{The Main Shortcomings of the Used Condoms are Revealed during their Operation in Everyday Life and in Physical Work}

a) Continuous use of conventional condoms, regardless of the use of a fixing device, from friction of the penis head against the bell wall (especially during physical work) leads to damage to the skin of the penis until the formation of open wounds, which leads to a temporary cessation of physical work and the use of condom until the healing of these wounds (calluses). b) The seam connecting the bell to the pipe (ending with the rim) proves to be very fragile, and the condom during operation is most often torn along this seam.

c) The use of condom without a fixing device in everyday life and in physical work is impossible, and the fastening device in the form of an adhesive tape or in the form of an adhesive layer on its inner wall cannot be used because of its strong adhesion to the skin of the penis and the impossibility of painlessly freeing it of it.

The aim of the invention is to exclude the possibility of mechanical damage to the penis by the walls of the condom, increase strength and lengthen the life of the condom, and increase the convenience of practical use of the condom.

\section{The Goal is Achieved by}

a) In the proposed condom, the bell is a spherical expansion of thickened rubber, whose diameter is much larger than the diameter of the penis, so that the head of the penis is inside this sphere and does not touch the walls of the condom.

b) There is no seam joining the spherical extension to the pipe. Instead, there is a smooth transition from a thick rubber sphere to a thin tube rubber.

c) To ensure that the urinal does not pull the condom from the penis, another plastic ring is put on the connecting plastic tube, with a diameter slightly larger than the diameter of the tube to which the cord is attached, of suitable length. The cord is attached to the waist, which is worn on the waist of the patient.

d) Fastening of the condom on the penis is carried out either with a rubber ring of suitable diameter, corresponding to the diameter of the penis, or a rope. When using a rubber ring, it is first worn on a condom, then the spherical part of the condom is worn on the penis, after which the ring is rolled onto the penis to the rim of the condom. Alternatively, the rubber ring is first rolled onto the penis, then the 


\section{Medicinal \& Analytical Chemistry International Journal}

spherical part of the condom is put on the penis, then the ring is rolled over the rim of the spherical part of the condom.

If you use a rope, then first put on the spherical part of the condom on the penis, then just below the rim wrap the cord with a rope and tie a rope to the bow. After the condom is fixed to the penis, in order that it does not spontaneously slip off the penis, the cord attached to the plastic ring is tied to the waistband that is worn on the waist of the patient. Belt is usually a ribbon about one centimeter wide. The exact size of all parts of the condom depends on the height of the man and the thickness of his penis, but can be made in three different ways, as in the "Urotzel" campaign.

A significant difference between the proposed condom is that, in order to exclude mechanical damage to the penis, instead of a part of the bell-shaped condom, a spherical expansion is made of thickened rubber of the appropriate diameter; in order to increase the service life of the condom, the seam connecting the spherical bell to the pipe is eliminated; for the convenience of using the condom, the fastener is secured by means of a rubber ring or a string that tightens around the penis, and in order that the urine collecting device does not pull the condom from the penis, a plastic ring is attached to the tube connecting them, through which a cord attached to the waist worn on the waist of the patient.

The main advantages of the proposed condom is the spherical shape of the bell of the condom, which excludes the possibility of mechanical damage to the penis by rubbing it against the walls of the condom for physical work. The absence of a seam on the condom allows longer life, and the use of a rubber ring or rope to fix the condom on the penis increases the ease of use of the condom and improves the quality of life of the patient.

The proposed uroprezervativ in conjunction with the urine sampler is designed for permanent wearing. At night, it is desirable to remove it. Approbation of the proposed condom was performed in 12 patients with incontinence after removal of the prostate adenoma at the age of $50-77$ years. Patients with a variety of professions continued to work in their workplaces, where they worked before the operation, at an air temperature of -30 to $+40 \mathrm{C}$. They were engineers with harmful working conditions, masons, concrete workers, agricultural workers. More than a year of observations, none of them had any diseases associated with the constant wearing of condom. It should be noted that in 2 patients who used similar condom (with rings), but in which there was no spherical expansion, during work requiring great mobility, corns could arise in hot weather, which required the temporary suspension of such work.

No accompanying erection and, moreover, priapism in patients with constant wearing of condom prevented Erection could occur only in those cases when a corresponding intimate situation was created.

\section{Claim}

Male uroprezervativ, characterized by the fact that in order to exclude the possibility of mechanical damage to the penis by the walls of the condom, to increase the strength and lengthen the life of the condom, to increase the convenience of practical use, use a condom with a spherical extension without a joint, and fixing the condom with a rubber ring or rope and ring on the tube connecting it with the urinal.

\section{Essay}

The invention relates to the field of medical technology and can be used in urology in the treatment of patients suffering from any type of urinary incontinence. The aim of the invention is to exclude the possibility of mechanical damage to the penis by the walls of the condom, increase strength and lengthen the life of the condom, and increase the convenience of practical use of the condom. The proposed condom has a spherical extension into which the head of the penis sinks, which excludes the possibility of contact with the walls of the condom. The absence of a joint increases the service life of the product, and the fixing of the condom with the help of a rubber ring or a rope, as well as a ring on the tube connecting the condom with the urine collecting device, increases the ease of operation of the condom. 